\title{
Virtual Geographic Environments in Socio-Environmental Modeling: A fancy distraction or a key to communication?
}

\author{
Alexey Voinov ${ }^{1}$, Arzu Çöltekin ${ }^{2}$, Min Chen $^{3}$, Ghassan Beydoun ${ }^{4}$
}

${ }^{1}$ Faculty of Geoscience and Earth Observation (ITC), University of Twente, P.O. Box 217, 7500 AE Enschede, The Netherlands, +31 67365 7124, aavoinov@gmail.com, orcid.org/0000-0002-2985-4574

${ }^{2}$ Department of Geography, University of Zurich, Winterthurerstrasse 190, 8057, Zurich, Switzerland, +41-44-6355440, arzu.coltekin@geo.uzh.ch, orcid.org/0000-0002-3178-3509

${ }^{3}$ Key Laboratory of Virtual Geographic Environment (Ministry of Education of PRC), Nanjing Normal University , 210023, Nanjing, China, +86 13951760902, chenmin0902@163.com

${ }^{4}$ Faculty of Engineering and IT, University of Technology Sydney, PO Box 123 Broadway NSW 2007, Australia, ghassan.beydoun@uts.edu.au

\begin{abstract}
Modeling and simulation are recognized as effective tools for management and decision support across various disciplines; however, poor communication of results to end users is a major obstacle for properly using and understanding model output. Visualizations can play an essential role in making modeling results accessible for management and decision-making. Virtual Reality (VR), and the VR implementation in Geography-related domains known as Virtual Geographic Environments (VGEs) are popular and potentially very rewarding ways to visualize socio-environmental models. However, using them properly in the context of system modeling is still a challenge. There is a fundamental conflict between abstraction and realism: models are always goal-driven, and created to simplify reality and to focus on certain crucial aspects of the system; while VR, by definition, attempts to replicate reality as closely as possible, and has often been technology-driven. While there are benefits to showing things as they are, this elevated realism may add to the complexity curse in modeling, and the message might be diluted by too many (background) details. This conflict between 'the desire to show a phenomenon with high fidelity' versus 'losing information because of too much detail' is linked to the concepts of information overload and cognitive load. Moreover, modeling is always associated with the treatment of uncertainty - something we do need to keep in mind when projecting model results into a virtual reality. In this paper, we examine the use of VR and, specifically, VGEs in socio-environmental modeling based on the literature, and discuss how VGEs and simulation modeling can be married in a mutually beneficial way that makes VGEs more effective for users, at the same time broadening the usage of simulation modeling to new problems.
\end{abstract}

\section{Keywords}

Complexity, uncertainty, virtual reality, stakeholders, cognition, users 


\section{Introduction}

Virtual Geographic Environments (VGEs) are a type of web- and computer-based geographic visualizations built by merging geographic knowledge, virtual reality technology, network technology, and geographic information technology with the objective of providing enhanced understanding and experiencing of the physical world. They allow users to 'feel the environments in person' by means of stimulating the senses and creating the feeling of presence, to explore the environments 'beyond reality' by simulating geographic phenomena and supporting collaborative geographic experiments (Chen, Lin, and Lu 2017). In addition to geographic information technologies, VGEs have been largely powered by the developments in Virtual Reality (VR) and related domains, for example, by the achievements of the computer gaming and animation industries. As such, they already use substantial amounts of dynamic modeling to describe the movement of objects and to implement various visualization techniques. However, more recently, there is a growing interest in using socio-environmental models in the context of VGE (Lin et al. 2013; Lin, Chen, and Lu 2013; Lin et al. 2015; Chen et al. 2013, 2015). Socioenvironmental models are models employed for the simulation of environmental processes and interactions between societal and environmental systems (Voinov et al. 2014). As VGEs seek realism, they need to mimic reality as closely as possible including the dynamic features that change with time. This desire to represent an evolving reality leads to increasing interest in process-based modeling, which can improve the background for actions that take place in the VGEs. Process-based models go beyond empirical interpretations of available data and also use theoretical knowledge about the processes involved, as well as information generated from previous studies of analogous systems. This makes them more realistic for extrapolation beyond the scope of the data sets available.

Socio-environmental modeling has been developing over the past $40+$ years as a useful tool for decision support, communication and understanding of system dynamics. Models have been developed for various systems at different scales, from global - e.g. Global Climatic Models (Dickinson 1984; Semenov and Stratonovitch 2010), to local - e.g. models of fishponds (Svirezhev, Krysanova, and Voinov 1984), predator prey interactions (Voinov 2008), bushfires (Plucinski et al. 2017), floods (Xie et al. 2017) or 'smart cities' (Kramers et al. 2014). Some of these spatio-temporal models can certainly be useful for an improved representation of the physical environment in VGEs including both the physical, ecological and social features (say, display how vegetation grows as we progress through seasons, or how areas get flooded in case of a major storm, or how cities grow with their populations). Representing such spatiotemporal aspects of the real world can potentially make a VGE feel more real and immersive to its users.

Even more promising are the powers of VGEs that can be used to enhance the communicative function of some models. While communication of knowledge is certainly one of the main purposes of modeling (Voinov 2008), it has been recognized that communicating model output to end users, especially to nonprofessionals or even researchers from different domains, is a challenging task (Lee et al. 2016). The context for each user is different and each is often concerned with only a specific part of a model rather than all of it. Usually models tell their stories in text, tables and graphics. Identifying effective ways to enable 'zooming in' on the relevant parts of the model would be of huge benefit to its users and to the knowledge sharing function of any model. Indeed, the ambition behind using a VGE is that by placing the model results in a visual form that can be 'naturally consumed' by humans, we create powerful interactive tools to assist, or even drive decision-making and human behavior. However, it is yet to be explored how we can do this properly, making sure that VR indeed helps convey the message rather than distract stakeholders from the science, and analyses that come out of the modeling exercises.

In this conceptual paper, we reflect on the possible synergies between process-based systems models and VGEs that we may want to develop in the future.

\section{Modeling and complexity}

Since the term 'modeling' has been vastly overused in the scientific literature, let us first clarify some pertinent definitions to avoid any misunderstanding. Here we define a model in the most general sense as a simplification, thus an abstraction of reality (Batty and Torrens 2005). Be it a conceptual/statistical/ mathematical or a graphic/visual model (e.g. a cartographic map or a 3D city model), the purpose of modeling is to create an abstract representation that focuses on what is most important for the purpose of 
its creation, that is, to answer a particular question or to meet the needs of a particular project (Voinov 2008). Throughout this paper, the word modeling refers to numerical modeling when in context of spatiotemporal modeling or when used as a standalone term, whereas it also refers to scientific visualization when it is used in context of 3D models or 3D modeling.

As simplifications of reality, models cannot and should not include as many details as possible. The art of modeling is about determining the most important elements, interactions, processes and factors. Human beings seek to simplify and abstract the reality, because most phenomena in the real world are too complex to comprehend. While creating a model, we focus on particular elements and features of reality that we find important for the purpose we have in mind. By definition, models are designed to focus only on some aspects of reality. As a result, obviously no model is a perfect representation of reality. Box's (1976) well-known saying that "all models are wrong but some are useful" is a concise expression of this.

This may sound somewhat contrary to some efforts in domains such as physics or engineering, where the goal of modeling is usually to include as many details about the studied system as possible. A common argument is that it is only the lack of data, information or computational power that stops us from doing so. For example, in engineering, a big step in simplification might take place inadvertently when we decide on the object of our analysis: once we decide that it is a bridge that we are modeling, anything that is not relevant to that bridge will be excluded from the analysis. With this 'simplification', arguably we can afford examining more details about that bridge.

As opposed to the engineering context such as the one presented above, in socio-environmental modeling, we deal with systems that are open, evolving and adapting; and as such they are not clearly defined and separated from their environment in space and time. We model these systems to improve our understanding of processes and phenomena involved, and to analyze the extreme scenarios of what is possible and what can happen (e.g. extreme natural hazards such as floods or landslides, or extreme socio-economic situations like epidemics or market crashes or changes in subsidies and taxes). Complexity of the systems becomes an obstacle to our understanding. Incorporating too much detail and realism in a socio-environmental model can lead to what is known as the Bonnini paradox: "A model is built in order to achieve understanding of an observed causal process, and the model is stated as being a simulation program in order that the assumptions and functional relations may be as complex and realistic as possible. The resulting program produces outputs resembling those observed in the real world, and inspires confidence that the real causal process has been accurately represented. However, because the assumptions incorporated in the model are complex and their mutual interdependencies are obscure, the simulation program is no easier to understand than the real process was." (Starbuck and Dutton 1971, 14)

An important distinction here is gathering as much information as possible (which, in most cases, is a good idea), versus including all the information in a representation such as a simulation model or a visualization (which, in most cases, is likely to be a bad idea). The desire to account for a multitude of possible features of the phenomena or object of interest is not surprising, given that we always strive for more realism. But human cognition has limitations: for example, it is well documented that the human working memory can handle only a limited number of 'objects' (or bits of information); more precisely we can process 4-7 objects at a time (Miller 1956; Cowan 2000).

Dealing with system complexity is probably the biggest challenge in the art of modeling. Certain details about a system could be very important to define its current and future states, and we are likely to misrepresent the system evolution if we fail to include such details. Furthermore, the importance of various system components may also change with time and space: what is unimportant today may become crucial tomorrow, and vice versa. So, on the one hand, there is always a desire (and thus, a tendency) to make the model more complex, and to include as many details as possible.

On the other hand, every new variable, process, relationship or parameter added to the model comes with additional uncertainty and ambiguity. As Oreskes $(2003,20)$ puts it: "A complex model may be more realistic yet at the same time more uncertain". Complex models are hard to calibrate, hard to test, and hard to communicate. As a result, they are hard to trust, and thus are easily dismissed for, apparently, being "uncertain" and therefore unreliable. This is what we have witnessed with climate change 
simulations, when state-of-the-art Global Climate Models were criticized and ignored (Idso, Carter, Singer 2016). They were labeled as 'too uncertain' and therefore inappropriate for management and policymaking.

There is much complaint that we are living in an increasingly complex world, which naturally requires more complexity in the models. Tainter (1988) describes how human societies gain complexity as they develop, which eventually brings them to a collapse. But is it really that the world is becoming more complex or is it just that we are trying to understand and capture more complexity? Arbesman $(2016,13)$ claims that "We are in a new era, one in which we are building systems that can't be grasped in their totality or held in mind of a single person; they are simply too complex". But this is something we do by choice. This brings us back to the Bonnini paradox. The world has always been infinitely complex. An individual human organism, with all the cells, tissues, fluids, organs, bacteria, viruses, interacting and constantly changing and adapting is probably no less complex than the whole society that assumes humans as entities that interact within; and environmental systems that interact with each other as well as with humans. While human created systems (social systems) indeed become increasingly complex (think of cities or the Internet), this does not mean that the world itself is becoming more complex. Complexity has always been there; it is only that we are daring to consider more complexity in our models, given that we can capture and computationally process more of it. In other words, it is very much up to us to decide how much complexity we need in our projects, and how much of it we can afford to deal with. The level of complexity we allow is defined by the purpose of modeling.

We do not want to complain about complexity in our models. Complexity is by choice. According to the famous saying attributed to Einstein, "If you can't explain it simply, you don't understand it yourself." If we revert to complexity in our models, this is probably due to a lack of understanding. In fact, it is easier to build a complex model than a simple one. However by allowing more complexity, we also defeat the very purpose of modeling, which is simplification.

Still, we need to clarify that by recognizing the complexity paradox in modeling, by no means do we want to say that only simple models are relevant and that we should ignore the overwhelming complexity of our world. We would agree with Ostrom, Janssen, and Anderies $(2007,15176)$ who caution against assuming "that all problems of resource governance can be represented by a small set of simple models". Analysis and decisions are not going to be simple, and should not rely on models that make false or oversimplified assumptions about the systems. But, as discussed earlier, making models overly complex is not going to be a solution as well, because of mounting uncertainty and loss of model controllability ('Bonnini paradox').

We build models to manage complexity, not to ignore it. There are several ways to deal with complexity, besides simplifying the model itself.

First, there is the model integration approach (Laniak et al 2013), when we learn to couple existing, previously designed components into new models. This does not automatically solve the problem of increasing complexity of models (Voinov and Cerco 2010), but it does allow us to build a hierarchy of models of various complexity levels and gives us a way to navigate through these different levels, as needed to address the different purposes of modeling. A complex modeling task can be decomposed into various small or simple modeling efforts, enabling different model builders to focus on their specific fields and develop well-tested atomic models of high quality.

Secondly, by engaging stakeholders in the modeling process in the so-called participatory modeling exercises (Voinov et al. 2016). Stakeholders are groups of people who can impact the system we analyze or who may be impacted by it; they are the ones who have a 'stake' in that system. By bringing them into the modeling process we can introduce some safeguards to avoid going beyond the understanding and needs of these target user groups. Model users, stakeholders, in this case also become model developers and they also learn from the modeling process, and guide the modelers to the type of models that suit their needs best. During the modeling process, different stakeholders from different domains or disciplines can negotiate with each other, and thus engage in a joint effort that fully considers the diverse knowledge background, expertise levels, abilities, and other use requirements. Analyzing user needs and requirements, and moderating user participation as well as properly assessing the outcomes from 
observational or controlled user studies require careful deliberation and established procedures (e.g., Coolican 1990; Courage and Baxter 2005; Martin 2007). A naively conducted user study might lead to misguided decisions.

Thirdly, we can better cope with complexity when we have appropriate tools to process, interpret and visualize models output. It has been shown that model output can be extremely complex and it requires additional efforts to make sense of it and to present it in ways coherent and useful to stakeholders (Lee et al., 2016). Often the output of models comes as numerous binary or decimal digits, which are hard to understand and use, especially for non-professional users, stakeholders, and decision-makers. Special efforts are needed to translate these results into meaningful graphics (e.g., tendency charts, statistical graphs, and 2D or 3D static or animated maps) or tables (e.g., hash tables or statistical tables) to better support communication and understanding.

As we discuss below, all these approaches are relevant for the interface between modeling and VGE. $2 \mathrm{D} / 3 \mathrm{D}$ visualizations, or VR displays are the next generation of approaches that can be instrumental in translating the complex output of complex models into comprehensible, compelling scenarios and powerful stories, which, in turn, can further contribute to parameter adjustment, comparison of results and improved decision-making and management. However this will work only if we can properly manage the complexity of the VR visualizations themselves, making them user-specific (personalized), scalable and modular.

\section{VGE for modeling}

We believe that there are at least two ways how VGEs can be very useful for modeling purposes.

First, realistic visualizations (such as VGEs) can be useful tools to engage users (Çöltekin, Pettit, and Wu 2015; van Lammeren et al. 2010), and collect corresponding user data for modeling and exploring. For example, user behavior can be explored utilizing VGEs as visualization tools to learn how they interpret and understand visual information about the environment and how they react to this information (e.g., Lokka and Çöltekin 2016). In this case we can control the conditions in which we conduct the experiment. For example, in a wayfinding experiment, we can provide all participants with the same environmental conditions: same weather, same lighting, same traffic, stable temperature etc. Similarly we can use fictional VGEs and control for the possible effects of familiarity. Being able to control such additional factors allows for experimental control, and thus helps avoiding confounding variables.

We can track human behavior and choices when reacting to situations created in the VR and learn how this information is used to make decisions (e.g., Regian, Shebilske, and Monk 1992; Bliss, Tidwell, and Guest 1997; Van Dam, Laidlaw, and Simpson 2002; Lin, Chen, and Lu 2013). Such knowledge acquired in VR experiments can then be used in our models to better describe the behavior of agents. For example, in agent based modeling, interaction agent models can be used to describe what messages agents need to exchange whilst collaborating on a specific goal. Agents as autonomous entities are particularly good enablers to model human interactions, and at the same time they facilitate separating tasks of modeling of the environment from tasks of modeling the agents' actions and interactions. In this sense, VR can be used to elicit information about human behavior. This information obtained in VR experiments can also be useful for scoping the most relevant parts of the model of the environment in which the agents/humans are situated. This leads to the second utility of VGE.

In line with the 'engagement' argument presented above, the second utility of VGEs as such is that, as powerful visualization tools, they can improve the communication of modeling results to the public. In other words, the consequences of a policy or environmental phenomenon can be simulated and presented in such a way that the non-experts (including the policy makers, who often rely on summary reports) would quickly comprehend their impact. For example, a VGE for hydrology modeling can be reused by disaster management responders or by population groups affected by floods; the dynamic visual scenario can be built based on these modeling results and thus even non-professional users can get the idea of what will happen. A set of agent based models describing the behavior of responders can be coupled with such a VGE to examine effectiveness of their actions in protecting public assets. Another set of agent based models describing population movement coupled with the same hydrology modeling 
VGE can be instead used to assess self evacuation timeliness to shelters as water levels rise (e.g., Li et al. 2012). More examples can be found in similar evacuation studies for other disasters (e.g., fire and earth earthquake) (e.g.,Torrens 2015a, 2015b, 2017; Li et al. 2014, 2015). On the other hand, because such a visualization may have quite a dramatic effect, the designer should be careful not to overwhelm and mislead the user, and the viewer should be able to view such a visualization critically (Çöltekin, Lokka, and Boer 2015).

Similarly, such use of VGEs can help in transdisciplinary teams to communicate across disciplinary boundaries and find clearer understanding of the processes and systems involved. A VGE can be designed as a workspace to enable experts from different fields to share, reuse, integrate and implement socio-environmental models (Lin, Chen, and Lu 2013). It would thus serve for the developing of dynamic virtual scenarios and processes. For example, when a meteorologist and a hydrologist conduct joint simulations, VGEs can facilitate their discussion and experiments. The idea that the VR can be used for collaborative projects has been proposed along with the early instances of VR (e.g., Billinghurst, Weghorst, and Furness 1998; Goodchild, 2009); however, the technology has progressed rapidly in the last decades, and the potential of VR for collaborative work is possibly more realistic now than ever before. In such an environment, interdisciplinary experts can discuss and communicate remotely and distributedly through the network, looking at the provided synchronous visual and vivid virtual scenes that they can experience or perceive in their daily life, rather than exploring highly specialized disciplinary figures or tables, which may not be always obvious and may hinder common understanding and collaborative research (e.g., Zhu et al. 2007; Lin et al. 2010; Xu et al. 2011; Chen et al. 2012). The users can explore diverse modeling strategies, scenarios or simulation schemes according to their different preferences and priorities, and compare the results that follow. Similarly, the decision makers can be involved, immediately sharing and explaining their requirements and values, potentially leading to more informed negotiations, consensus and improved management.

While all of the above sounds very promising, such propositions have not yet been rigorously verified in proper, controlled user experiments, at least not at a level that would allow generalized statements. A VGE might help in some cases, while it might impair the user performance in other cases. This has been shown in some experimental studies using realistic displays, and named 'naive realism'. The users preferred the realistic displays even though their performance was better with the simplified (more abstract) visualizations (Smallman and St. John 2005). Replications of this finding are rare with VGEs (Lokka and Çöltekin 2017), however they should not be overlooked.

We see that there is a big contradiction between the main goals of modeling and VGEs. A main goal of VGEs has been to provide as much realism as possible (Lin and Batty 2009). The goal of VR, as the name suggests, has been to develop representations that would make presence in the virtual world almost impossible to distinguish from real life (Sherman and Craig 2002). Immersion and telepresence (thus realism) was about including reality as it is, and then adding even more to it. For example, a fundamental definition of virtual reality by MacEachren et al. (1999) proposes "four l's".r immersion, interactivity, information intensity and intelligence of objects. Thus, the basic premise of the VR concept has indeed been such that the more details could be included to the virtual representations and the closer their appearance would match the human interpretations of reality, the better.

This desire to "mimic reality" as an inherent feature in the VGEs is quite contrary to what modeling is about. As discussed earlier, modeling by definition is about simplification. If a VGE is to enhance the modeling toolkit, then it should also be selective of the details that it presents to the public. For example, if we are trying to assist in directing the public to shelters, the focus could be on roads and lifelines leading to the shelters, and may be less detailed when it comes to present the texture of buildings or surrounding landscape features. We may also consider providing the aerial perspective in appropriate moments to to put more emphasis on the travel route (which gives a better overview than a first-person perspective), or combine multiple perspectives when it might enhance the comprehension and improve learning. In this case one might argue that we will be compromising one of the fundamental ideas of VR, as we would be projecting the 3D reality onto a 2D map. However, in a goal-driven system, this should not be a problem as long as our goal is better met. We do not want realism and VR to become a selfserving goal on their own. 
VGEs should be made adaptive to focus on the message that the model delivers. We argue that in many cases too much realism in VGEs and too much detail can be detrimental for the message that we try to convey with the model (e.g., Smallman and John 2005). As with any visualization tool, VGEs have to be stripped of the unnecessary details to package the model results in a compelling way, taking into account the user community addressed (e.g., Lokka and Çöltekin 2017). For example, evacuees need only to be informed about the water levels and flow on the roads leading to shelters. Identifying the required details for the user community is akin to solving the 'framing problem' for each community of users. Note that, attempts to model the extent of relevance of particular details have been made in GIScience, but this is a complex problem in itself with many dimensions (e.g., Sabbata and Reichenbacher 2012).

The frame problem from Artificial Intelligence $(\mathrm{Al})$ is identifying the required parameters for an autonomous agent (robot) to execute their actions in a given environment (McCarthy and Hayes 1969). In other words, for VGEs to become effective, interfaces and built-in mechanisms are required to hide and show, on a need basis, parameters from the environment to the stakeholders. Of course, the challenge is discerning the needed parameters. In Al, this is often addressed by installing additional semantics (knowledge) to the parameters or providing learning mechanisms to expand the set of identified parameters as deemed necessary by the modelers (Dennett 2006). In VGEs, a human presence is anticipated as VGEs are in essence an IT system interface. Keeping this functional aspect of VGEs in mind, they can be enhanced to invite human contributions. It may well be enough to use such enhancement to a VGE to elicit human input when a decision with the provided parameters is deemed as 'risky' or 'uncertain'. For example, as discussed earlier, human presence coupled with a VR interface can provide additional behavioral information that can be used to determine the context of model usage (the same way a GUI window requests additional input when a mouse action seems spurious - e.g. a click on the ' $x$ ' box on the top right).

Yet another challenge is the visualization of uncertainty (e.g., MacEachren et al., 2005). We are used to a single unique reality. But models produce ranges of outputs. How do we control the uncertainty caused by model integration? How do we present the uncertain model output in VGEs? When placing the model output in the context of VR, we may be creating a false impression that we know how the system will look, and how it will be changing. In fact, we would need to be able to add some 'fuzziness' in the VR that we are projecting. If we are using a VGE, for example, to demonstrate the extent of flooding that is output from a hydrology model, it would be wrong to present the results as exact areas and locations flooded. We need tools to demonstrate the possible extremes and perhaps their likelihoods (risk), as well as ranges of such extremes as generated by models. It appears that some form of 'fuzziness' in the visualization, implicitly conveys uncertainty, though the source of uncertainty is not easy to communicate. For example, it has been shown that people trust that the more realistic the visual models are, the better the data quality is (Zanola, Fabricant, and Çöltekin 2009), suggesting that one might convey uncertainties in the data by modifying the visualization design (e.g., using fuzzy edges, blur, etc.). However, this is not quite the same as presenting the ranges or interval estimates that models often produce, and further thinking on visualization methods and empirical testing is necessary to find effective ways to visualize uncertainty inherent in models.

While there is a growing body of new technologies for VGEs in modeling (e.g., Wen et al. 2013; Zhang et al. 2016), to date, little research has explored the contradiction between complexity and simplicity in spatio-temporal systems modeling and VGEs. The richness of information and data taken into account, often seen as a plus in science and engineering, does not immediately constitute an advantage in modeling, neither it is always a benefit in VGE. To the contrary, it acts as a detriment to the central functionality of a VGE, that being a friendlier IT interface. Added complexity clouds the ability of humans to reason effectively and quickly - when most needed (e.g. in emergency situations). Finding the 'sweet spot' between complexity and ease of use is yet to be investigated sufficiently to enable a systematic design of VGEs, especially when linked to simulation models.

\section{Discussion and conclusions}

At this point, the benefits and drawbacks of integrating VGEs and spatio-temporal modeling are not fully established, empirical studies on visualization provide mixed evidence regarding the advantages of 3D, 
and we see that 3D is not always better that 2D (Shepherd 2008). There are contradicting findings about whether some situations are better served with a 3D interface, and if so, which kind of 3D, under what design choices and/or in relation to which tasks and which user groups (Çöltekin, Lokka, and Zahne 2016). The question becomes even more complex when we bring in the fourth dimension (time) and interactivity as in a VGE. For example, it remains yet to be seen to what extent the 4D visualizations that are offered by VGEs are useful to communicate modeling results to the stakeholders. Visualizing temporal phenomena is a challenge in itself (also beyond VGEs). There is some evidence that animations, for example, might not work as expected (e.g., see the literature review section presented by Russo et al. 2014).

After all, the success of the human mind has been strongly associated with its ability to select what is most important in its perception of reality (Anderson 1991). When we look at a forest we want to see the forest, not the trees. There is only so much information that an ordinary human brain can effectively process (Miller 1956; Cowan 2000). By providing too much detail, and consuming the brain with processing these details we may only be distracting our users from the main message. These human abilities in processing details and their preferences on choosing the details to focus on are also very individual. For example, it is reported that as much as $20 \%$ of the population is not able to see in stereo (Ware 2004). It is important to remember that virtual reality is not defined solely by stereoscopic visualizations. According to various authors it is defined by several criteria; such as the above mentioned MacEachren et al.'s (1990) "four Is": immersion, interactivity, information intensity and intelligence of objects. These "four Is" do not depend on stereoscopic viewing, even though stereoscopic viewing might increase the sense of presence and enhance the precision of spatial perception for certain tasks (Mclntire, Having, and Geiselman 2014). Besides stereoscopic vision, however, 3D perception depends on many different depth cues (for a recent review see Reichelt et al., 2010). There is even evidence from, neurophysiological research that we collect visual information only in $2 \mathrm{D}$, and it is our brains that transform the input into 3D visualizations to match our environment (Finlayson, et al., 2017). This also means that we all may be doing this transformation in slightly different ways, indeed adding subjectivity to our interpretation of the third dimension. When we add the fourth dimension, time, the results might get even more ambiguous. We are attached to our perceptions, which may be illusions or may be very real but in any case are largely driven by our background knowledge, our vested interests, cultural biases, and own values (Voinov et al. 2014; Glynn et al., 2017). (Fig.1).

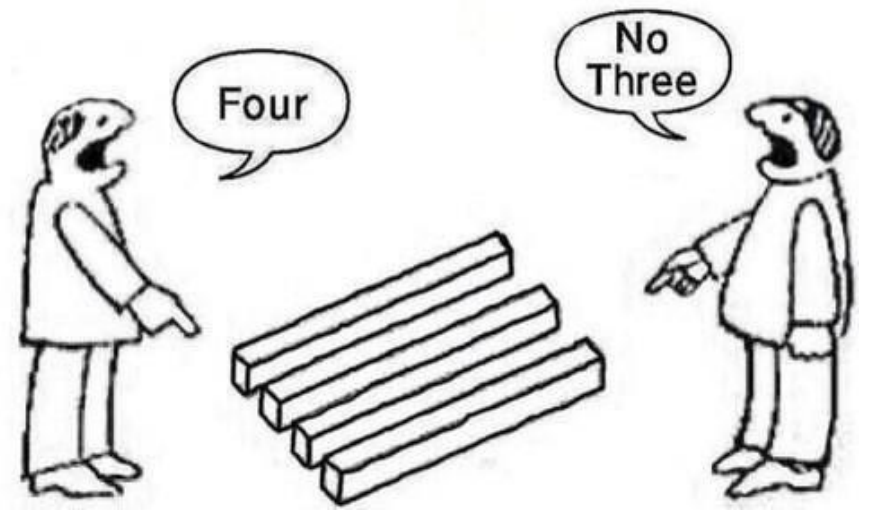

Fig.1. The way people see things can be crucial for their decisions. It speaks to their System 1 thinking and may be very difficult to change. (https://plus.google.com/103488786418978849574/posts/ 4r8nG6rFQfh)

VGEs appear to be good if the goal is to impress, attract, engage, and perhaps distract from reality, and 
to immerse us into virtual worlds (Chen, Lin, and Lu 2017; Çöltekin, Lokka, and Zahne 2016). How good are they to explain, to connect, to improve understanding and learning? These are subjects of spatial cognition research. Generalizable answers are yet to be established. Perhaps a marriage with modeling and the influence of some of the principles borrowed from complex systems analysis paradigms such as systems thinking, which advocates looking at systems in their entirety focusing on interactions and connections between system elements, can affect VGEs, and move them toward new goals and ideas.

John Hanke, Niantic CEO and developer of the PokemonGo app advocates for Augmented Reality (AR) instead of only VR: "My thing about VR is I'm afraid it can be too good, in the sense of being an experience that people want to spend a huge amount of time in" (Batchelor 2017). "In a VR situation, you're isolating yourself from everyone around you and entering this completely virtual space. AR is designed to add, enhance the things you do as a human being: Being outside, socializing with other people, shopping, playing, and having fun. AR can make all those things better" (Johnson 2016). Along these lines, we perhaps ought to explore some of the ways in which we can augment geographic environments instead of merely creating virtual ones, so that model output can be directly projected into the reality by adding certain features for people to see and comprehend, without taking them entirely into the virtual world.

Whether it is in AR or VR, just like models themselves, VGEs that are built to support modeling should be designed for specific purposes. In all cases, we should be clear what are these purposes, why are we doing this. What and for whom are the VGEs designed for? How are they going to be used? What are the elements of a model output that they are displaying, and how can they help us focus on what we think is most important in the model? Clearly, different users require different VGEs according to their different needs. Unless we are very clear how VGEs are to enhance our ability to understand and solve problems, we may only be building some self-serving tools. The reality created, instead of enhancing the model output and helping to make the right decisions, can overwhelm the human ability to properly understand the systems and to comprehend their evolution in time and space.

\section{References}

Anderson, J. R. 1991. "Is human cognition adaptive?" Behavioral and Brain Sciences, 14(03): 471-485. doi:10.1017/S0140525X00070801.

Arbesman, S. 2016. Overcomplicated: Technology at the Limits of Comprehension. New York: Penguin Random House.

Batty, M., and P.M. Torrens. 2005. "Modelling and prediction in a complex world." Futures, 37(7): 745766. doi:10.1016/j.futures.2004.11.003.

Batchelor, J. 2017. “John Hanke: VR is 'too good', could be a 'problem for society". Accessed Aug 7. http://www.gamesindustry.biz/articles/2017-04-04-john-hanke-vr-is-too-good-could-be-aproblem-for-society.

Billinghurst, M., S. Weghorst, and T. Furness. 1998. "Shared space: An augmented reality approach for computer supported collaborative work." Virtual Reality 3(1): 25-36.doi:10.1007/BF01409795.

Bliss, J. P., P. D. Tidwell, and M. A. Guest. 1997. "The effectiveness of virtual reality for administering spatial navigation training to firefighters." Presence: Teleoperators and Virtual Environments 6(1): 7386.doi: 10.1162/pres.1997.6.1.73.

Box, G. E. P. 1976." Science and Statistics." Journal of the American Statistical Association 71: 791-799. doi:10.1080/01621459.1976.10480949

Chen, M., H. Lin, and G. N. Lu. 2017. "Virtual Geographic Environments." In The International Encyclopedia of Geography, edited by American Association of Geographers. New Jersey: Wiley.doi: 10.1002/9781118786352.wbieg0448. 
Chen, M., H. Lin, O. Kolditz, and C. Chen. 2015. "Developing dynamic Virtual Geographic Environments (VGEs) for geographic research." Environmental Earth Sciences 74:6975-6980. doi: 10.1007/s12665015-4761-4.

Chen, M., H. Lin, M. Y. Hu, L. He, and C. X. Zhang. 2013. "Real-geographic-scenario-based virtual social environment: integrate geography with social research." Environment and planning B-Planning \& Design 40(6): 1103-1121. doi:10.1068/b38160.

Chen, M., H. Lin, Y. N. Wen, L. He, and M. Y. Hu. 2012. "Sino-VirtualMoon: A 3D web platform using Chang'e-1 data for collaborative research." Planetary and Space Science 65(1): 130-136. doi: 10.1016/j.pss.2012.01.005.

Coolican, H. 1990. Research methods and statistics in psychology. UK: Hodder \& Stoughton Educational.

Çöltekin, A., C. Pettit, and B. Wu. 2015. "Geovisual analytics: human factors." International Journal of Digital Earth 8(8), 595-598. doi:10.1080/17538947.2015.1047173

Çöltekin, A., I-E. Lokka, and A. Boer. 2015. "The utilization of publicly available map types by non-experts -- A choice experiment." Proceedings of the 27th International Cartographic Conference (ICC2015), London, 8-12.

Çöltekin, A., I. Lokka, and M. Zahner. 2016. "On the Usability and Usefulness of 3D (Geo) Visualizations A Focus on Virtual Reality Environments." Proceedings of International Archives of Photogrammetry, Remote Sensing and Spatial Information Sciences, Prague, 12-19.

Courage, C., and K. Baxter. 2005. Understanding your users: A practical guide to user requirements. Morgan Kaufmann. 1st edition. San Francisco: Morgan Kaufmann.

Cowan, N. 2000. "The magical number 4 in short-term memory: a reconsideration of mental storage capacity." The Behavioral and Brain Sciences, 24(1): 87-114.

Dennett, D. 2006. "Personal and sub-personal levels of explanation". In Philosophy of Psychology: Contemporary Readings, edited by Bermúdez, J. New York: Routledge.

Dickinson, R. E. 1984. "Modeling evapotranspiration for three-dimensional global climate models." In Climate Processes and Climate Sensitivity, Hansen, J. E., T. Takahashi, and R. E. Dickinson, eds. 5872.Washington D. C.: American Geophysical Union.

Finlayson, N. J., X. Zhang, and J. D. Golomb. 2017. "Differential Patterns of 2D Location versus Depth Decoding along the Visual Hierarchy." Neurolmage 147: 507-516.

doi:10.1016/j.neuroimage.2016.12.039.

Glynn, P.D, A.A Voinov, C.D Shapiro, and P.A White. 2017. "From Data to Decisions: Processing Information, Biases, and Beliefs for Improved Management of Natural Resources and Environments." Earth's Future. 5 (4): 356-78, doi:10.1002/2016EF000487.

Goodchild, M. F. 2009. "Virtual geographic environments as collective constructions." In Virtual geographic environments, Lin, H., and M. Batty, eds.,15-24. Beijing: Science Press.

layson, N. J., X. Zhang, and J. D. Golomb. 2017. "Differential Patterns of 2D Location versus Depth Decoding along the Visual Hierarchy." Neurolmage 147: 507-16. doi:10.1016/j.neuroimage.2016.12.039.

Idso, C.D., R.M. Carter, and S.F. Singer. 2016. "Why Scientists Disagree About Global Warming." NIPCC Report, $135 \mathrm{p}$.

Johnson, E. 2006. "Why Pokémon Go's John Hanke says augmented reality is better than virtual reality." Accessed Aug 7. https://www.recode.net/2016/9/19/12965508/pokemon-go-john-hankeaugmented-virtual-reality-ar-recode-decode-podcast 
Kramers, A., M. Höjer, N. Lövehagen, and J. Wangel. 2014. "Smart sustainable cities-Exploring ICT solutions for reduced energy use in cities." Environmental Modelling \& Software 56: 52-62.

doi:10.1016/j.envsoft.2013.12.019.

Laniak, G. F., G. Olchin, J. Goodall, A. Voinov, M. Hill, P. Glynn, G. Whelan, et al. 2013. "Integrated environmental modeling: A vision and roadmap for the future". Environmental Modelling \& Software 39:323. doi:10.1016/j.envsoft.2012.09.006.

Lee, J. S., T. Filatova, A. Ligmann-Zielinska, B. Hassani-Mahmooei, F. Stonedahl, I. Lorscheid, A. Voinov, et al. 2015. "The Complexities of Agent-Based Modeling Output Analysis." Journal of Artificial Societies and Social Simulation 18 (4): 1-27. doi:10.18564/jasss.2897.

Li, Y., J. H. Gong, J. Zhu, L. Ye, Y. Q. Song, and Y. J. Yue. 2012. "Efficient dam break flood simulation methods for developing a preliminary evacuation plan after the Wenchuan Earthquake." Natural Hazards and Earth system sciences 12:97-106. doi: 10.5194/nhess-12-97-2012.

Li, W. H., J. H. Gong, P. Yu, S. Shen, R. Li, and Q. S. Duan. 2014. "Simulation and analysis of individual trampling risk during escalator transfers." Physica A: Statistical Mechanics and its Applications 408: 119-133. doi: 10.1016/j.physa.2014.03.071.

Li, W. H., J. H. Gong, P. Yu, S. Shen, R. Li, and Q. S. Duan. 2015. "Simulation and analysis of congestion risk during escalator transfers using a modified social force model." Physica A: Statistical Mechanics and Its Applications 420: 28-40. doi: 10.1016/j.physa.2014.10.044.

Lin, H., M. Chen, G. N. Lu, Q. Zhu, J. H. Gong, X. You, Y. N. Wen, et al. 2013. "Virtual Geographic Environments (VGEs): A New Generation of Geographic Analysis Tool." Earth-Science Reviews 126: 7484. doi:10.1016/j.earscirev.2013.08.001.

Lin, H., and M. Batty. 2009. "Virtual geographic environments: a primer." In Virtual Geographic Environments, Lin, H., M. Batty, eds., 1-10. Beijing: Science Press.

Lin, H., Zhu, J., Gong, J.H., Xu, B. L., and H. Qi. 2010. "A grid-based collaborative Virtual Geographic Environment for the planning of silt dam systems." International Journal of Geographical Information Science 24 (4) 607-621.doi:10.1080/13658810903012425.

Lin, H., Chen, M., and G.N. Lu. 2013. "Virtual geographic environment: a workspace for computer-aided geographic experiments." Annals of the Association of American Geographers 103(3), 465-482.doi: 10.1080/00045608.2012.689234.

Lin, H., M. Batty, S. E. Jorgensen, B. J. Fu, M. Konecny, A. Voinov, P. Torrens, et al. 2015. "Virtual Environments Begin to Embrace Process-based Geographic Analysis." Transactions in GIS 19(4): 493498. doi:10.1111/tgis.12167.

Lokka, I.-E., and A. Çöltekin. 2016. "Simulating navigation with virtual 3D geovisualizations - A focus on memory related factors." Proceedings of the ISPRS - International Archives of the Photogrammetry, Remote Sensing and Spatial Information Sciences, Prague.

Lokka, I-E., and A. Çöltekin. 2017 . "Designing a memorable virtual 3D world: Empirical evidence for a texture-based highlighting approach." International Journal of Digital Earth, DOI:

10.1080/17538947.2017.1349842.

MacEachren, A. M., R. Edsall, D. Haug, R. Baxter, G. Otto, R. Masters, S. Fuhrmann, et al. 1999. "Exploring the potential of virtual environments for geographic visualization." Proceedings of the Annual Meeting of the Association of American Geographers, Honolulu, HI: 23-27.

MacEachren, A. M., A. Robinson, S. Hopper, S. Gardner, R. Murray, M. Gahegan , and E. Hetzler. 2005. 
"Visualizing geospatial information uncertainty: What we know and what we need to know." Cartography and Geographic Information Science, 32(3): 139-160.doi: 10.1559/1523040054738936.

Martin, D. W. 2007. Doing psychology experiments. Boston: Cengage Learning.

McCarthy, J., and P. J. Hayes. 1969. "Some philosophical problems from the standpoint of artificial intelligence". In Readings in artificial intelligence, Webber, B. L., and N. J. Nilsson, eds., 463-502. California: Morgan Kaufmann Publishers, Inc.

Mclntire, J. P., P. R. Havig, and E. E. Geiselman. 2014. "Stereoscopic 3D displays and human performance: A comprehensive review." Displays 35(1): 18-26. doi:10.1016/j.displa.2013.10.004.

Miller, G. A. 1956. "The magical number seven, plus or minus two: some limits on our capacity for processing information." Psychological Review 63(2): 81-97. doi: 10.1037/h0043158

Mortara, M., C. E. Catalano, F. Bellotti, G. Fiucci, M. Houry- Panchetti, and P. Petridis. 2013. "Learning cultural heritage by serious games." Journal of Cultural Heritage 15(3): 318-325. doi:

10.1016/j.culher.2013.04.004

Oreskes, N. 2003. "The role of quantitative models in science." In Models in Ecosystem Science, C. D. Canham, C. D., J. J. Cole, and W. K. Lauenroth, eds., 13-31. Princeton: Princeton University Press.

Ostrom, E., M. A. Janssen, and J. M. Anderies. 2007. "Going beyond Panaceas." Proceedings of the National Academy of Sciences of the United States of America 104 (39): 15176-78. doi:10.1073/pnas.0701886104.

Plucinski, M.P., A. L. Sullivan, C. J. Rucinski, and M. Prakash. 2017. "Improving the reliability and utility of operational bushfire behaviour predictions in Australian vegetation." Environmental Modelling \& Software, 91: 1-12. doi: 10.1016/j.envsoft.2017.01.019.

Reichelt, S., R. Häussler, G. Fütterer, and N. Leister. 2010. "Depth cues in human visual perception and their realization in 3D displays." Proceedings of The International Society for Optical Engineering, 7960. Orlando, 5.

Regian, J. W., W. L. Shebilske, and J. M. Monk. 1992. "Virtual reality: an instructional medium for visual spatial tasks." Journal of Communication, 42(4): 136-149. doi: 10.1111/j.1460-2466.1992.tb00815.x.

Russo, P., C. Pettit, A. Çöltekin, M. Imhof, M. Cox, and C. Bayliss. 2014. "Understanding soil acidification process using animation and text: An empirical user evaluation with eye tracking." In Cartography from Pole to Pole, Buchroithner, M., N. Prechtel, and D. Burghardt, eds., 431-448. Heidelberg: Springer-Verlag Berlin Heidelberg.

Sabbata, S. D., and T. Reichenbacher. 2012. "Criteria of geographic relevance: an experimental study." International Journal of Geographical Information Science, 26(8): 1495-1520. doi:

10.1080/13658816.2011.639303.

Semenov, M. A., and P. Stratonovitch. 2010. "Use of multi-model ensembles from global climate models for assessment of climate change impacts." Climate Research, 41(1): 1-14.doi: 10.3354/cr00836.

Shepherd, I. D. H. 2008. "Travails in the third dimension: a critical evaluation of three-dimensional geographical visualization.” In Geographic Visualization: Concepts, Tools and Applications, Dodge, M., M. McDerby, and M. Turner, eds., 199-210. Chichester, UK: Wiley.

Sherman, W. R., and A. B. Craig. 2002. Understanding virtual reality: Interface, application, and design. Elsevier.

Smallman, H. S., and M. John. 2005. "Naive realism: Limits of realism as a display principle." Proceedings of the Human Factors and Ergonomics Society Annual Meeting 49(17): 1564-1568. 
doi:10.1177/154193120504901714

Starbuck, W.H., and J. M. Dutton. 1971. "The history of simulation models." In Computer Simulation of Human Behavior, Dutton, J.M., and W.H. Starbuck, eds.,14. New Jersey: Wiley.

Svirezhev, Y. M., V. Krysanova, and A. Voinov. 1984. "Mathematical Modeling of a Fishpond Ecosystem." Ecological Modelling 21: 315-37. doi: 10.1016/0304-3800(84)90066-8.

Sword-Daniels, V., Ch. Eriksen, E. E Hudson-Doyle, R. Alaniz, C. Adler, T. Schenk, and S. Vallance. 2016. "Embodied Uncertainty: Living with Complexity and Natural Hazards." Journal of Risk Research 9877: 1-18. doi:10.1080/13669877.2016.1200659.

Tainter, Joseph A. 1988. The Collapse of complex Societies. Cambridge University Press.

Torrens, P. M. 2015a. "Slipstreaming human geosimulation in virtual geographic environments." Annals of GIS 21(4): 325-344. doi: 10.1080/19475683.2015.1009489.

Torrens, P. M. 2015b. "Intertwining agents and environments." Environmental Earth Sciences 74(10):7117-7131. doi: 10.1007/s12665-015-4738-3.

Torrens, P. M. 2017. "A computational sandbox with human automata for exploring perceived egress safety in urban damage scenarios." International Journal of Digital Earth, doi:10.1080/17538947.2017.1320594.

Van Dam, A., D. H. Laidlaw, R. M. Simpson. 2002. Experiments in immersive virtual reality for scientific visualization. Computers \& Graphics 26(4): 535-555.doi: 10.1016/S0097-8493(02)00113-9.

Van Lammeren, R., J. Houtkamp, S. Colijn, M. Hilferink, and A. Bouwman. 2010. Affective appraisal of 3D land use visualization. Computers, Environment and Urban Systems 34(6): 465-475.

doi:10.1016/j.compenvurbsys.2010.07.001.

Voinov, A. 2008. Systems Science and Modeling for Ecological Economics. US: Academic Press.

Voinov, A, and C. Cerco. 2010. "Model Integration and the Role of Data." Environmental Modelling \& Software 25 (8): 965-69. doi:10.1016/j.envsoft.2010.02.005.

Voinov, A., R. Seppelt, S. Reis, J.E.M.S. Nabel, and S. Shokravi. 2014. "Values in Socio-Environmental Modelling: Persuasion for Action or Excuse for Inaction." Environmental Modelling \& Software 53 (March): 207-212. doi:10.1016/j.envsoft.2013.12.005.

Voinov, A., N. Kolagani, M. K. McCall, P. D. Glynn, M. E. Kragt, F. O. Ostermann, S. A. Pierce, et al. 2016. "Modelling with Stakeholders - Next Generation." Environmental Modelling and Software 77: 196220. doi:10.1016/j.envsoft.2015.11.016.

Ware, C. 2004. Information Visualization: Perception for Design. (3rd ed.). San Francisco, CA, USA: Morgan Kaufmann.

Wen, Y., Chen, M., Lu, G. N., and H. Lin. 2013. "Prototyping an open environment for sharing geographical analysis models on cloud computing platform." International Journal of Digital Earth, 6(4):356-382. doi:10.1080/17538947.2012.716861.

Xie,J., H. Chen, Z. L. Liao, X. Y. Gu, D. J. Zhu, and J Zhang, 2017. "An integrated assessment of urban flooding mitigation strategies for robust decision making." Environmental Modelling \& Software 95:143155. doi:10.1016/j.envsoft.2017.06.027.

Xu, B. L., H. Lin, L.S. Chiu, Y. Hu, J. Zhu, M. Y. Hu, and W. N. Cui. 2011. "Collaborative Virtual Geographic Environments: a case study of air pollution simulation." The Information of the Science 181 (11): 2231-2246. doi: 10.1016/j.ins.2011.01.017. 
Zanola, S., S. I. Fabrikant, and A. Çöltekin. 2009. "The effect of realism on the confidence in spatial data quality in stereoscopic 3D displays." In Proceedings of the 24th international cartography conference (ICC 2009), Santiago, Chile.

Zhang, C. X., M. Chen, R. R. Li, C. Y. Fang, and H. Lin. 2016. "What's going on about geo-process modeling in virtual geographic environments (VGEs)." Ecological Modelling 319: 147-154.doi: 10.1016/j.ecolmodel.2015.04.023.

Zhu, J., J. H. Gong, W. G. Liu, T. Song, and J. Q. Zhang. 2007. "A collaborative Virtual Geographic environment based on P2P and grid technologies." The Information of the Science 177(21): 4621-4633. doi: $10.1016 /$ j.ins.2007.05.006. 